\title{
GREEN FUNCTION OF TRIHARMONIC HEAT EQUATION USING ADDITION FORMULA OF FUNCTION $e^{-x^{3}}$
}

\author{
A. ANSARI \\ Received 19 October, 2016
}

\begin{abstract}
In this paper, using the Mellin transform of Airy function we present an integral addition formula for the function $e^{-x^{3}}$. In deriving this representation, we make use of the Hankel functions of first kind and apply this representation to get the Green function of triharmonic heat equation in terms of the Airy functions.
\end{abstract}

2010 Mathematics Subject Classification: 33C10; 34B27; 35K30

Keywords: Green function, triharmonic heat equation, Mellin transform, Airy function

\section{INTRODUCTION}

As we know for the harmonic heat equation

$$
\frac{\partial}{\partial t} u(x, y, t)=\Delta u(x, y, t), \quad t>0, x, y \in \mathbb{R}, \quad u(x, y, 0)=\delta(x) \delta(y),
$$

where $\Delta=\frac{\partial^{2}}{\partial x^{2}}+\frac{\partial^{2}}{\partial y^{2}}$ is the Laplacian operator, the associated Green function (fundamental solution) is given by [17]

$$
u(x, y, t)=\frac{1}{2 \sqrt{\pi t}} e^{-\frac{x^{2}+y^{2}}{4 t}} .
$$

Also, the Green function of biharmonic heat equation [5]

$$
\frac{\partial}{\partial t} u(x, y, t)+\Delta^{2} u(x, y, t)=0, \quad t>0, x, y \in \mathbb{R}, \quad u(x, y, 0)=\delta(x) \delta(y),
$$

has been recently presented in terms of the quartic Lévy stable function given by $[18,23]$

$$
L_{4}(\xi, t)=\frac{1}{\pi} \int_{0}^{\infty} e^{-t r^{4}} \cos (r \xi) d r .
$$

This function is the Green function of generalized heat equation

$$
\frac{\partial}{\partial t} u(x, t)+\frac{\partial^{4}}{\partial x^{4}} u(x, t)=0, \quad t>0, x \in \mathbb{R}, \quad u(x, 0)=\delta(x),
$$


related to the following fractional exponential operator

$$
u(x, t)=e^{-t \frac{\partial^{4}}{\partial x^{4}}} \delta(x)=L_{4}(x, t) .
$$

The main strategy of obtaining the Green function of biharmonic heat equation is applying the two dimensional fractional exponential operator

$$
u(x, y, t)=e^{-t \Delta^{2}} \delta(x) \delta(y)=e^{-t\left[\frac{\partial^{2}}{\partial x^{2}}+\frac{\partial^{2}}{\partial y^{2}}\right]^{2}} \delta(x) \delta(y),
$$

which can be extended for the higher order harmonic heat equations. This is our main motivation for showing the Green function of triharmonic heat equation

$$
\frac{\partial}{\partial t} u(x, y, t)+\Delta^{3} u(x, y, t)=0, \quad t>0, x, y \in \mathbb{R}, \quad u(x, y, 0)=\delta(x) \delta(y),
$$

which its solution can be consequently derived as

$$
u(x, y, t)=e^{-t \Delta^{3}} \delta(x) \delta(y) .
$$

For obtaining the above solution which has been much less developed in the literature, see for example [26], first we get an integral addition formula for the function $e^{-x^{3}}$. Next, we present the fundamental solution in terms of the Airy functions.

In this sense, we organize the paper as follows. In Section 2, we recall the definitions of the gamma and reciprocal gamma functions along with its triplication formula. We also mention some algebraic and operational properties of the Airy functions of first and second kinds.

In Section 3, we state an integral relation between the function $e^{-x^{3}}$ and Airy function on the well-known Hankel contour and then we construct an integral addition formula for function $e^{-x^{3}}$. The obtained addition formula is given in terms of the imaginary parts of products of Airy functions and can be simplified with respect the products of Hankel functions of first kind.

In Section 4, using the exponential differential operators we get the Green function of triharmonic heat equation (1.9) in terms of the Airy functions.

\section{PRELIMINARIES}

\subsection{The Gamma functions}

The gamma function and the reciprocal gamma function are defined as

$$
\begin{gathered}
\Gamma(s)=\int_{0}^{\infty} e^{-\tau} \tau^{s-1} d \tau, \quad \Re(s)>0, \\
\frac{1}{\Gamma(s)}=\frac{1}{2 \pi i} \int_{\mathrm{Ha}} e^{\tau} \tau^{-s} d \tau,
\end{gathered}
$$

where Ha is the Hankel contour in complex plane with a cut along the negative real semi-axis $\arg \tau=\pi$ and consisting of the following three parts

(1) $\arg \tau=\pi, \quad|\tau| \geq \epsilon$, 
(2) $-\pi \leq \arg \tau \leq \pi, \quad|\tau|=\epsilon$,

(3) $\arg \tau=-\pi, \quad|\tau| \geq \epsilon$.

For this function, the well-known triplication formula is given by [1, p. 256, 6.1.19]

$$
\Gamma(s)=\frac{1}{2 \pi} 3^{s-\frac{1}{2}} \Gamma\left(\frac{s}{3}\right) \Gamma\left(\frac{s+1}{3}\right) \Gamma\left(\frac{s+2}{3}\right), \quad \Re(s)>0 .
$$

\subsection{The Airy functions}

The classical Airy functions of first and second kinds $\operatorname{Ai}(x)$ and $\operatorname{Bi}(x)$ are defined as $[3,6,9,12,20,27]$

$$
\begin{gathered}
\operatorname{Ai}(x)=\frac{1}{\pi} \int_{0}^{\infty} \cos \left(x t+\frac{t^{3}}{3}\right) d t, \\
\operatorname{Bi}(x)=\frac{1}{\pi} \int_{0}^{\infty}\left(e^{x t-\frac{t^{3}}{3}}+\sin \left(x t+\frac{t^{3}}{3}\right)\right) d t,
\end{gathered}
$$

and the Mellin transform of function $\operatorname{Ai}(x)$ is given by [27, p. 96]

$$
\mathcal{M}\{\operatorname{Ai}(x) ; s\}=\int_{0}^{\infty} x^{s-1} \operatorname{Ai}(x) d x=3^{-(s+2) / 3} \frac{\Gamma(s)}{\Gamma\left(\frac{s+2}{3}\right)}, \quad \Re(s)>0 .
$$

Also, for $|\arg (a)|<\frac{\pi}{3}$ and $|\arg (b)|<\frac{\pi}{3}$ some integral addition formula have been derived in the literature, see $[12,25]$

$$
\begin{gathered}
\operatorname{Ai}(a+b)=\int_{0}^{1} \operatorname{Ai}^{\prime}\left(\frac{a}{\xi^{\frac{1}{3}}}\right) \operatorname{Ai}^{\prime}\left(\frac{b}{(1-\xi)^{\frac{1}{3}}}\right) \frac{d \xi}{\xi^{\frac{2}{3}}(1-\xi)^{\frac{2}{3}}}, \\
\operatorname{Ai}(a+b)=\frac{a b}{a+b} \int_{0}^{1} \operatorname{Ai}\left(\frac{a}{\xi^{\frac{1}{3}}}\right) \operatorname{Ai}\left(\frac{b}{(1-\xi)^{\frac{1}{3}}}\right) \frac{d \xi}{\xi^{\frac{4}{3}}(1-\xi)^{\frac{4}{3}}} .
\end{gathered}
$$

Moreover, the following identities hold for the Airy functions of first and second kinds [27, p. 8], [1, p. 447, 10.4.28]

$$
\begin{gathered}
\operatorname{Ai}^{\prime}\left(x e^{-\frac{\pi i}{3}}\right)=\operatorname{Ai}^{\prime}\left(-x e^{\frac{2 \pi i}{3}}\right)=\frac{e^{-\frac{\pi i}{3}}}{2}\left[\operatorname{Ai}^{\prime}(-x)-i \operatorname{Bi}^{\prime}(-x)\right], \\
\operatorname{Ai}^{\prime}(-x)-i \operatorname{Bi}^{\prime}(-x)=e^{-\frac{\pi i}{6}} \frac{x}{\sqrt{3}} H_{2 / 3}^{(1)}\left(\frac{2}{3} x^{\frac{3}{2}}\right)
\end{gathered}
$$

where the Hankel function of first kind $H_{v}^{(1)}(x)$ is defined as [19, p. 914, 8.421(1)]

$$
H_{v}^{(1)}(x)=\frac{2 e^{-\frac{v \pi i}{2}}}{\pi i} \int_{-\infty}^{\infty} e^{i x \cosh (u)-v u} d u, \quad-1<\Re(v)<1, x>0 .
$$




\section{InTEgral AdDition Formula}

Lemma 1. For $\Re(s)>0$, the Mellin transform of function $e^{-x^{3}}$ is given by

$$
\begin{aligned}
\mathcal{M}\left\{e^{-x^{3}} ; s\right\} & =2 \pi\left[3^{-(4 s+1) / 6} \frac{1}{\Gamma\left(\frac{s+1}{3}\right)}\right]\left[3^{-(s+2) / 3} \frac{\Gamma(s)}{\Gamma\left(\frac{s+2}{3}\right)}\right], \\
& =2 \pi\left[3^{-(4 s+1) / 6} \frac{1}{\Gamma\left(\frac{s+1}{3}\right)}\right] \mathcal{M}\{\operatorname{Ai}(x) ; s\} .
\end{aligned}
$$

Proof. According to [19, p. 1131], for the Mellin transform of the function $e^{-x^{3}}$

$$
\mathcal{M}\left\{e^{-x^{3}} ; s\right\}=\frac{1}{3} \Gamma\left(\frac{s}{3}\right),
$$

and taking into account the relations (2.3) and (2.6), we get the result.

Lemma 2. The following representation holds for the function $e^{-x^{3}}$ and the Airy function

$$
e^{-x^{3}}=-i 3^{-1 / 6} \int_{\mathrm{Ha}} e^{\tau} \tau^{-1 / 3} \operatorname{Ai}\left(3^{2 / 3} \tau^{1 / 3} x\right) d \tau,
$$

where Ha is the Hankel contour.

Proof. Taking into account the relation (2.2) for the reciprocal gamma function $\frac{1}{\Gamma\left(\frac{s+1}{3}\right)}$ and considering the representation (3.1), we can present a simple comparison between the Mellin transform of the Airy function and $e^{-x^{3}}$. After a little algebra and using the following property of Mellin transform

$$
\mathcal{M}\{f(a x) ; s\}=\frac{1}{a^{s}} F(s), \quad a>0,
$$

we obtain the result.

Theorem 1. For $|\arg (a)|<\frac{\pi}{3}$ and $|\arg (b)|<\frac{\pi}{3}$, the following integral addition formulas hold

$$
\begin{aligned}
& e^{-(a+b)^{3}}=\frac{2}{3^{1 / 6}} \int_{0}^{1} \int_{0}^{\infty} \frac{e^{-\tau}}{\sqrt[3]{\tau}} \Im\left[e^{\frac{\pi i}{3}} \operatorname{Ai}^{\prime}\left(\frac{3^{2 / 3} \tau^{1 / 3} e^{-\frac{\pi i}{3}} a}{\xi^{1 / 3}}\right) \mathrm{Ai}^{\prime}\left(\frac{3^{2 / 3} \tau^{1 / 3} e^{-\frac{\pi i}{3}} b}{(1-\xi)^{1 / 3}}\right)\right] \\
& \times \frac{1}{\xi^{\frac{2}{3}}(1-\xi)^{\frac{2}{3}}} d \tau d \xi \\
& e^{-(a+b)^{3}}=2 \sqrt{3} \frac{a b}{a+b} \int_{0}^{1} \int_{0}^{\infty} e^{-\tau \Im}\left[\operatorname{Ai}\left(\frac{3^{2 / 3} \tau^{1 / 3} e^{-\frac{\pi i}{3}} a}{\xi^{1 / 3}}\right) \operatorname{Ai}\left(\frac{3^{2 / 3} \tau^{1 / 3} e^{-\frac{\pi i}{3}} b}{(1-\xi)^{1 / 3}}\right)\right] \\
& \times \frac{1}{\xi^{\frac{4}{3}}(1-\xi)^{\frac{4}{3}}} d \tau d \xi \text {. }
\end{aligned}
$$


Proof. First, we use the relation (3.3) to present an addition formula for the function $e^{-x^{3}}$ with respect to the addition formula of Airy function. Next, we compute the remaining integral on the Hankel contour in three parts by applying Titchmarsh theorem for the inverse Laplace transforms of multi-valued functions [8, 10,21]

$$
\begin{aligned}
e^{-(a+b)^{3}}= & -i 3^{-1 / 6} \int_{0}^{1} \int_{\mathrm{Ha}}\left[e^{\tau} \tau^{-1 / 3} \mathrm{Ai}^{\prime}\left(\frac{3^{2 / 3} \tau^{1 / 3} a}{\xi^{1 / 3}}\right) \mathrm{Ai}^{\prime}\left(\frac{3^{2 / 3} \tau^{1 / 3} b}{(1-\xi)^{1 / 3}}\right)\right] \\
& \times \frac{1}{\xi^{\frac{2}{3}}(1-\xi)^{\frac{2}{3}}} d \tau d \xi \\
= & -i 3^{-1 / 6} \int_{0}^{1} \int_{\infty}^{0}\left[e^{\tau} \tau^{-1 / 3} \mathrm{Ai}^{\prime}\left(\frac{3^{2 / 3} \tau^{1 / 3} a}{\xi^{1 / 3}}\right) \mathrm{Ai}^{\prime}\left(\frac{3^{2 / 3} \tau^{1 / 3} b}{(1-\xi)^{1 / 3}}\right)\right]_{\tau=r e^{i \pi}} \\
& \times \frac{1}{\xi^{\frac{2}{3}}(1-\xi)^{\frac{2}{3}}} d \tau d \xi \\
& -i 3^{-1 / 6} \int_{0}^{1} \int_{\pi}^{-\pi}\left[e^{\tau} \tau^{-1 / 3} \mathrm{Ai}^{\prime}\left(\frac{3^{2 / 3} \tau^{1 / 3} a}{\xi^{1 / 3}}\right) \mathrm{Ai}^{\prime}\left(\frac{3^{2 / 3} \tau^{1 / 3} b}{(1-\xi)^{1 / 3}}\right)\right]_{\tau=\epsilon e^{i \theta}} \\
& \times \frac{1}{\xi^{\frac{2}{3}}(1-\xi)^{\frac{2}{3}}} d \tau d \xi \\
& -i 3^{-1 / 6} \int_{0}^{1} \int_{0}^{\infty}\left[e^{\tau} \tau^{-1 / 3} \mathrm{Ai}^{\prime}\left(\frac{3^{2 / 3} \tau^{1 / 3} a}{\xi^{1 / 3}}\right) \mathrm{Ai}^{\prime}\left(\frac{3^{2 / 3} \tau^{1 / 3} b}{(1-\xi)^{1 / 3}}\right)\right]_{\tau=r e^{-i \pi}} \\
& \times \frac{1}{\xi^{\frac{2}{3}}(1-\xi)^{\frac{2}{3}}} d \tau d \xi
\end{aligned}
$$

Taking $\epsilon \rightarrow 0$, we obtain the addition formula of function $e^{-x^{3}}$ in terms of the imaginary part of products of Airy functions as mentioned in (3.5). The result (3.6) is obtained by the same procedure.

Remark 1. For simplicity of the imaginary part of products of Airy functions in (3.5), we employ the relations (2.9) and (2.10), respectively, and present two representations as follows

$$
\begin{aligned}
\Im & {\left[e^{\frac{\pi i}{3}} \operatorname{Ai}^{\prime}\left(\frac{3^{2 / 3} \tau^{1 / 3} e^{-\frac{\pi i}{3}} a}{\xi^{1 / 3}}\right) \operatorname{Ai}^{\prime}\left(\frac{3^{2 / 3} \tau^{1 / 3} e^{-\frac{\pi i}{3}} b}{(1-\xi)^{1 / 3}}\right)\right] } \\
= & -\frac{1}{4}\left[\operatorname{Ai}^{\prime}\left(-\frac{3^{2 / 3} \tau^{1 / 3} a}{\xi 1^{1 / 3}}\right) \mathrm{Bi}^{\prime}\left(-\frac{3^{2 / 3} \tau^{1 / 3} b}{(1-\xi)^{1 / 3}}\right)+\mathrm{Bi}^{\prime}\left(-\frac{3^{2 / 3} \tau^{1 / 3} a}{\xi^{1 / 3}}\right) \mathrm{Ai}^{\prime}\left(-\frac{3^{2 / 3} \tau^{1 / 3} b}{(1-\xi)^{1 / 3}}\right)\right] \\
& -\frac{\sqrt{3}}{4}\left[\mathrm{Ai}^{\prime}\left(-\frac{3^{2 / 3} \tau^{1 / 3} a}{\xi^{1 / 3}}\right) \mathrm{Ai}^{\prime}\left(-\frac{3^{2 / 3} \tau^{1 / 3} b}{(1-\xi)^{1 / 3}}\right)-\mathrm{Bi}^{\prime}\left(-\frac{3^{2 / 3} \tau^{1 / 3} a}{\xi^{1 / 3}}\right) \mathrm{Bi}^{\prime}\left(-\frac{3^{2 / 3} \tau^{1 / 3} b}{(1-\xi)^{1 / 3}}\right)\right],
\end{aligned}
$$




$$
\begin{aligned}
& \Im\left[e^{\frac{\pi i}{3}} \operatorname{Ai}^{\prime}\left(\frac{3^{2 / 3} \tau^{1 / 3} e^{-\frac{\pi i}{3}} a}{\xi^{1 / 3}}\right) \operatorname{Ai}^{\prime}\left(\frac{3^{2 / 3} \tau^{1 / 3} e^{-\frac{\pi i}{3}} b}{(1-\xi)^{1 / 3}}\right)\right]= \\
& \quad-\frac{1}{8} \frac{3^{5 / 6} \tau^{2 / 3} a b}{\xi^{1 / 3}(1-\xi)^{1 / 3}} H_{v}^{(1)}\left(\frac{2}{3^{1 / 2}} \frac{\tau^{1 / 2} a^{3 / 2}}{\xi^{1 / 2}}\right) H_{v}^{(1)}\left(\frac{2}{3^{1 / 2}} \frac{\tau^{1 / 2} b^{3 / 2}}{(1-\xi)^{1 / 2}}\right)
\end{aligned}
$$

\section{THE GREEN FUNCTION OF TRIHARMONIC HEAT EQUATION}

In this section, using the exponential differential operators and the obtained addition formula in previous section, we intend to obtain the Green function of triharmonic heat equation in terms of the Airy functions. For knowing more applications of the exponential differential operators in the applied mathematics see $[2,4,7,11$, 13-16, 22, 24].

At the beginning, we show a fundamental lemma for the Green function of generalized heat equation of third order and state a theorem for the Green function of triharmonic heat equation. Although the obtained solution in very formal, but it presents an analytical form for the structure of Green function.

Lemma 3 ([7, 11]). The Green function of generalized heat equation

$$
\frac{\partial}{\partial t} u(x, t)=\frac{\partial^{3}}{\partial x^{3}} u(x, t), \quad t>0, x \in \mathbb{R}, \quad u(x, 0)=\delta(x),
$$

is obtained by applying the following exponential operator on the Dirac delta function

$$
u(x, t)=e^{t \frac{\partial^{3}}{\partial x^{3}}} \delta(x)=\frac{1}{\sqrt[3]{t}} \operatorname{Ai}\left(-\frac{x}{\sqrt[3]{t}}\right) .
$$

Theorem 2. The Green function of triharmonic heat equation

$$
\frac{\partial}{\partial t} u(x, y, t)+\Delta^{3} u(x, y, t)=0, \quad t>0, x, y \in \mathbb{R}, \quad u(x, y, 0)=\delta(x) \delta(y),
$$

is given by

$$
\begin{aligned}
u(x, y, t)= & -\frac{x y e^{-\frac{\pi i}{3}}}{2^{4 / 3} \pi^{2}} \int_{0}^{1} \int_{0}^{\infty} \int_{-\infty}^{\infty} \int_{-\infty}^{\infty} \frac{e^{-\tau} \tau^{-1 / 3}}{\xi^{2 / 3}(1-\xi)^{2 / 3}} \frac{e^{-2(u+v) / 3}}{\sqrt[3]{\cosh ^{2}(u) \cosh ^{2}(v)}} \\
& \times \operatorname{Ai}\left(-\frac{x \sqrt[6]{\frac{3 \xi}{t \tau}} e^{-\frac{\pi i}{6}}}{\sqrt[3]{2 \cosh (u)}}\right) \operatorname{Ai}\left(-\frac{y \sqrt[6]{\frac{3(1-\xi)}{t \tau}} e^{-\frac{\pi i}{6}}}{\sqrt[3]{2 \cosh (v)}}\right) d u d v d \tau d \xi .
\end{aligned}
$$

Proof. We begin with the following formal solution of triharmonic heat equation

$$
u(x, y, t)=e^{-t \Delta^{3}} \delta(x) \delta(y)=e^{-t\left[\frac{\partial^{2}}{\partial x^{2}}+\frac{\partial^{2}}{\partial y^{2}}\right]^{3}} \delta(x) \delta(y),
$$


and set $a=\sqrt[3]{t} \frac{\partial^{2}}{\partial x^{2}}$ and $b=\sqrt[3]{t} \frac{\partial^{2}}{\partial y^{2}}$ in relation (3.5) to get the solution as

$$
\begin{aligned}
e^{-t\left[\frac{\partial^{2}}{\partial x^{2}}+\frac{\partial^{2}}{\partial y^{2}}\right]^{3}} \delta(x) \delta(y)= & -\frac{a b}{4 \times 3^{2 / 3}} \int_{0}^{1} \int_{0}^{\infty} e^{-\tau} \sqrt[3]{\tau} \frac{1}{\xi(1-\xi)} \\
& \times\left[H_{2 / 3}^{(1)}\left(\frac{2}{3^{1 / 2}} \frac{t^{1 / 2} \tau^{1 / 2} \frac{\partial^{3}}{\partial x^{3}}}{\xi^{1 / 2}}\right) \delta(x)\right] \\
& \times\left[H_{2 / 3}^{(1)}\left(\frac{2}{3^{1 / 2}} \frac{t^{1 / 2} \tau^{1 / 2} \frac{\partial^{3}}{\partial y^{3}}}{(1-\xi)^{1 / 2}}\right) \delta(y)\right] d \tau d \xi .
\end{aligned}
$$

We now apply the integral representation (2.11) and identity (4.1), and simplify the above solution using the following relations

$$
\begin{aligned}
{\left[H_{2 / 3}^{(1)}\left(\frac{2}{3^{1 / 2}} \frac{t^{1 / 2} \tau^{1 / 2} \frac{\partial^{3}}{\partial x^{3}}}{\xi^{1 / 2}}\right) \delta(x)\right]=} & \frac{2 e^{-\frac{\pi i}{3}}}{\pi i} \int_{-\infty}^{\infty} e^{2 i \sqrt{\frac{\tau \tau}{3 \xi}} \cosh (u) \frac{\partial^{3}}{\partial x^{3}}-2 u / 3} \delta(x) d u \\
= & -\frac{2}{\pi} \int_{-\infty}^{\infty} e^{-2 u / 3} \frac{\sqrt[6]{\frac{3 \xi}{t \tau}}}{\sqrt[3]{2 \cosh (u)}} \\
& \times \operatorname{Ai}\left(-\frac{\left.x \sqrt[6]{\frac{3 \xi}{t \tau} e^{-\frac{\pi i}{6}}}\right) d u}{\sqrt[3]{2 \cosh (u)}}\right) \\
{\left[H_{2 / 3}^{(1)}\left(\frac{2}{3^{1 / 2}} \frac{t^{1 / 2} \tau^{1 / 2} \frac{\partial^{3}}{\partial y^{3}}}{(1-\xi)^{1 / 2}}\right) \delta(y)\right]=} & \frac{2 e^{-\frac{\pi i}{3}}}{\pi i} \int_{-\infty}^{\infty} e^{2 i \sqrt{\frac{t \tau}{3 \xi}} \cosh (v) \frac{\partial^{3}}{\partial y^{3}}-2 v / 3} \delta(y) d v, \\
= & -\frac{2}{\pi} \int_{-\infty}^{\infty} e^{-2 v / 3} \frac{\sqrt[6]{\frac{3(1-\xi)}{t \tau}}}{\sqrt[3]{2 \cosh (v)}} \\
& \times \operatorname{Ai}\left(-\frac{y \sqrt[6]{\frac{3(1-\xi)}{t \tau}} e^{-\frac{\pi i}{6}}}{\sqrt[3]{2 \cosh (v)}) d v} .\right.
\end{aligned}
$$

Finally, by substituting the above relations into (4.5) we obtain the result.

\section{REFERENCES}

[1] M. Abramowitz and I. A. Stegun, Handbook of mathematical functions with formulas, graphs, and mathematical tables. 9th printing, Dover, New York: John Wiley \& Sons., 1972. 
[2] A. Ansari, "Fractional exponential operators and nonlinear partial fractional differential equations in the Weyl fractional derivatives." Appl. Math. Comput., vol. 220, pp. 149-154, 2013, doi: https://doi.org/10.1016/j.amc.2013.05.066.

[3] A. Ansari, “ The fractional Airy transform." Hacet. J. Math. Stat., vol. 44, no. 4, pp. 761-766, 2015, doi: 10.15672/HJMS.2015449440.

[4] A. Ansari, "Some inverse fractional Legendre transforms of gamma function form." Kodai Math. J., vol. 38, no. 3, pp. 658-671, 2015, doi: 10.2996/kmj/1446210600.

[5] A. Ansari, "Remarks on the green function of space-fractional biharmonic heat equation using Ramanujan's master theorem." Kuwait J. Sci., vol. 44, no. 4, pp. 1-8, 2017.

[6] A. Ansari and M. Masomi, "Some results involving integral transforms of Airy functions.” Integral Transforms Spec. Funct., vol. 26, no. 7, pp. 539-549, 2015, doi: https://doi.org/10.1080/10652469.2015.1025072.

[7] A. Ansari, M. Ahamadi Darani, and M. Moradi, "On fractional Mittag-Leffler operators." Rep. Math. Phys., vol. 70, no. 1, pp. 119-131, 2012, doi: https://doi.org/10.1016/S00344877(13)60017-8.

[8] A. Ansari and A. Ansari, “ Solving partial fractional differential equations using the $\mathscr{L}_{A}$-transform." Asian-Eur. J. Math., vol. 3, no. 2, pp. 209-220, 2010, doi: $10.1142 / \mathrm{S} 1793557110000143$.

[9] A. Ansari and M. R. Masomi, "Some integral identities for products of Airy functions using integral transforms." Asian-Eur. J. Math., vol. 11, no. 1, p. 1850046, 2018, doi: 10.1142/S1793557118500468.

[10] A. Ansari and A. Refahi Sheikhani, "New identities for the Wright and the Mittag-Leffler functions using the Laplace transform." Asian-Eur. J. Math., vol. 7, no. 3, p. 1450038, 2014, doi: 10.1142/S1793557114500387.

[11] A. Ansari, A. Refahi Sheikhani, and S. Kordrostami, "On the generating function $e^{x t+y \phi(t)}$ and its fractional calculus." Cent. Eur. J. Phys., vol. 11, no. 10, pp. 1457-1462, 2013, doi: 10.2478/s11534-013-0195-3.

[12] H. Askari and A. Ansari, "Airy functions and Riesz fractional trigonometric operators." Integral Transforms Spec. Funct., vol. 29, no. 8, pp. 585-604, 2018, doi: https://doi.org/10.1080/10652469.2018.1472593.

[13] G. Dattoli, P. L. Ottaviani, and L. Vazquez, “Evolution operators equations: integration with algebric and finite difference methods. Applications to physical problems in classical and quantum mechanics and quantum field theory." Riv. Nuovo Cimento., vol. 20, pp. 1-133, 1997, doi: 10.1007/BF02907529.

[14] G. Dattoli, P. E. Ricci, and D. Sacchetti, "Generalized shift operators and pseudopolynomials of fractional order." Appl. Math. Comput., vol. 141, pp. 215-224, 2003, doi: https://doi.org/10.1016/S0096-3003(02)00334-X.

[15] G. Dattoli, P. E. Ricci, and I. Khomasuridze, " Operational methods, special polynomial and functions and solution of partial differential equations." Integral Transforms Spec. Funct., vol. 15, pp. 309-321, 2004, doi: https://doi.org/10.1080/10652460410001673013.

[16] G. Dattoli, H. M. Srivastava, and K. Zhukovsky, " Operational methods and differential equations with applications to initial value problems.” Appl. Math. Comput., vol. 184, pp. 979-1001, 2007, doi: https://doi.org/10.1016/j.amc.2006.07.001.

[17] L. Debnath and D. Bhatta, Integral transforms and their applications. New York: second ed., Chapman \& Hall/CRC, Taylor \& Francis Group, 2007.

[18] K. Górska, A. Horzela, K. A. Penson, and G. Dattoli, “The higher-order heat-type equations via signed Lévy stable and generalized Airy functions." J. Phys. A: Math. Theor., vol. 46, p. 16 pages, 2013, doi: 10.1088/1751-8113/46/42/425001. 
[19] I. S. Gradshteyn and I. M. Ryzhik, Table of integrals, series and products. New York: Seventh Edition, Academic Press, 2007.

[20] L. Moslehi and A. Ansari, "Integral representations of products of Airy functions related to fractional calculus.” J. Class. Anal., vol. 7, no. 2, pp. 99-112, 2015, doi: dx.doi.org/10.7153/jca-0710.

[21] L. Moslehi and A. Ansari, "Some remarks on inverse Laplace transforms involving conjugate branch points with applications." U. P. B. Sci. Bull. Series A-Appl. Math. Phys., vol. 78, no. 3, pp. 107-118, 2016.

[22] L. Moslehi and A. Ansari, "Squared radial Ornstein-Uhlenbeck processes and inverse Laplace transforms of products of confluent hypergeometric functions." Hacet. J. Math. Stat., 2016, doi: 10.15672/HJMS.20174622761.

[23] L. Moslehi and A. Ansari, "Integral representations of products of quartic Lévy stable functions." Integral Transforms Spec. Funct., vol. 28, no. 11, pp. 1-12, 2017, doi: https://doi.org/10.1080/10652469.2017.1376192.

[24] L. Moslehi and A. Ansari, "On M-Wright transforms and time-fractional diffusion equations." Integral Transforms Spec. Funct., vol. 28, no. 2, pp. 113-129, 2017, doi: https://doi.org/10.1080/10652469.2016.1252763.

[25] N. V. Proskurin, "Integral addition formulae for Airy function." Integral Transforms Spec. Funct., vol. 5, no. 1, pp. 143-146, 1997, doi: https://doi.org/10.1080/10652469708819130.

[26] W. Satsanit, "Solution to the triharmonic heat equation." Electron. J. Differ. Equ., no. 4, pp. 1-9, 2011.

[27] O. Vallee and M. Soares, Airy functions and applications to physics. London: Imperial College Press, 2004.

Author's address

A. Ansari

Department of Applied Mathematics, Faculty of Mathematical Sciences, Shahrekord University, P.O. Box 115, Shahrekord, Iran

E-mail address: alireza_1038@yahoo.com 